\title{
RADIAL DISTRIBUTION OF METEORIC PARTICLES IN INTERPLANETARY SPACE 5.6
}

\author{
John W. Rhee \\ Rose-Hulman Institute of Technology \\ Terre Haute, Indiana 47803 \\ and \\ Goddard Space Flight Center \\ Greenbelt, Maryland 20771
}

An attempt has been made to derive heliocentric distribution of meteoric particles in interplanetary space. Poynting-Robertson effect, collision process, and cometary dust injection have been included in this study. Three different radial distribution functions for different sizes have been derived. The number density appears to depend on particle size.

A series of important experiments has been carried out to obtain some information about the heliocentric distribution of cosmic dust particles which produce zodiacal light (Alexander, 1962; Alexander et al., 1965; Rhee et al., 1974; Humes et al., 1974). Recently Southworth and Sekanina (1973) studied the distribution of meteoroids in the solar system from the orbits of approximately 20,000 radar meteors observed in 1969. They reached the surprising new conclusion that the space density in ecliptic plane was minimum near $0.7 \sim 0.8 \mathrm{AU}$ and maximum in the asteroid belt, between 2 and $3 \mathrm{AU}$. The zodiacal light data obtained by Hanner et al., (1974) from Pioneer 10 drops off quite rapidly between 2.4 AU and 3.2 AU. There appears to be no zodiacal light beyond 3.3 AU. In the following we shall attempt to derive heliocentric distribution functions on a number of simple and plausible physical arguments.

(a) Poynting-Robertson Effect

It is a well-known fact that meteoric particles lose angular momentum in interplanetary space and spiral into the sun. If the Poynting-Robertson effect operates alone in interplanetary space, the particle number density $N$ must satisfy

$$
\left(\frac{\mathrm{dN}}{\mathrm{dr}}\right)_{\mathrm{p}-\mathrm{R}}=\left(\frac{\mathrm{dN}}{\mathrm{dt}}\right)_{\mathrm{p}-\mathrm{R}}\left(\frac{\mathrm{dt}}{\mathrm{dr}}\right)_{\mathrm{p}-\mathrm{R}}=-\left(\frac{\mathrm{N}}{\mathrm{r}}\right)
$$

which has a solution of $\mathrm{N} \sim \mathrm{r}^{-1}$ and $\mathrm{r}$ is the distance.

(b) Inter-Particle Collision Process

Southworth and Sekanina has shown that due to collisions N must satisfy

$$
\left(\frac{\mathrm{dN}}{\mathrm{dr}}\right)_{\mathrm{c}}=\left(\frac{\mathrm{dN}}{\mathrm{dt}}\right)_{\mathrm{c}}\left(\frac{\mathrm{dt}}{\mathrm{dr}}\right)_{\mathrm{p}-\mathrm{R}}=\mathrm{AN}^{2} \sqrt{\mathrm{r}}
$$

where $A$ is a constant. The exact solution of Equation 2 is $N \sim r^{-1.5}$. 


\section{(c) Cometary Particle Injection}

Since Poynting-Robertson effect and collision theory cannot explain the recent observational data from Pioneer 10, it is proposed that direct cometary injection will have to be taken into account. This might be done as

$$
\left(\frac{d N}{d r}\right)_{i}=\left(\frac{d N}{d t}\right)_{i}\left(\frac{d t}{d r}\right)_{p-R}
$$

where $\left(\frac{\mathrm{dN}}{\mathrm{d} t}\right)_{\mathrm{i}}$ represents cometary particle injection rate. The dust emission rate from comets is usually assumed to vary with $\mathrm{r}$ as $\mathrm{r}^{-\alpha}$ where $\alpha$ usually ranges from 0.5 to 5 (Sekanina, 1974). It is not clear whether cometary brightness is directly related to particle injection rate or not. Cometary brightness depends also on $\mathrm{r}$ as $\mathrm{r}^{-\beta}$ where $\beta$ typically ranges from 2 to 6 . It is quite possible that most of dust emitted by comets does not remain in the solar system due to solar radiation pressure. At any rate Equation 3 can be written as

$$
\left(\frac{\mathrm{dN}}{\mathrm{dr}}\right)_{\mathrm{i}}=-\beta \mathrm{r}^{-\mathrm{p}}
$$

where $\beta$ and $\mathrm{p}$ are constants.

(d) Southworth-Sekanina Equation as follows:

Following Southworth and Sekanina (1973), we shall expand $\frac{d N}{d r}$ in terms of $\left(\frac{d r}{d t}\right)_{p-R}$

$$
\frac{d N}{d r}=\sum_{i=1}^{3}\left(\frac{d N}{d t}\right)_{i}\left(\frac{d t}{d r}\right)_{p-R}=-\frac{N}{r}+A N^{2} \sqrt{r}-\beta r^{-p}
$$

Defining $\mathrm{y}=\mathrm{N}^{-1}$, Equation 5 can be rewritten as

$$
\frac{d y}{d r}-\frac{y}{r}=-A \sqrt{r}+\beta y^{2} r^{-p}
$$

The following three approximate specific solutions of Equation 6 have been derived

Case (a):

$$
\begin{gathered}
\mathrm{p}=0, \quad \mathrm{y}=\mathrm{A}_{1} \mathrm{r}-\mathrm{A}_{2} \mathrm{r}^{3 / 2}+\mathrm{A}_{3} \mathrm{r}^{4} \\
\frac{\mathrm{N}}{\mathrm{N}_{0}}=\frac{0.23}{\mathrm{r}-0.87 \mathrm{r}^{3 / 2}+0.1 \mathrm{r}^{4}}
\end{gathered}
$$

Case (b):

$$
p=1, \quad y=A_{4} r-A_{5} r^{3 / 2}+A_{6} r^{3}
$$

$$
\frac{N}{N_{0}}=\frac{0.25}{r-0.8 r^{3 / 2}+0.05 r^{3}}
$$

Case (c):

$$
\begin{gathered}
\mathrm{p}=2, \quad \mathrm{y}=\mathrm{A}_{7} \mathrm{r}-\mathrm{A}_{8} \mathrm{r}^{3 / 2}+\mathrm{A}_{9} \mathrm{r}^{2} \\
\frac{\mathrm{N}}{\mathrm{N}_{0}}=\frac{0.58}{\mathrm{r}-0.45 \mathrm{r}^{3 / 2}+0.03 \mathrm{r}^{2}}
\end{gathered}
$$


where $\mathrm{N}_{0}$ is the number density at $1 \mathrm{AU}$ and $\mathrm{A}_{1} \ldots \mathrm{A}_{9}$ are constants. These three cases represent cometary injection rates of $r^{-1}, r^{-2}$, and $r^{-3}$, respectively. A cometary injection rate of $r^{-4}$ leads to an unphysical situation and must be discarded under this approximation.

Case (a) has been fitted with Pioneer 8 and Pioneer 9 data (Rhee et al., 1974) and the normalized number density is represented by Equation 7. Equation 7 is also shown in Figure 1. An examination of Figure 1 shows that for $\mathrm{r}<0.4 \mathrm{AU}, \mathrm{N} \sim \mathrm{r}^{-1}$ and for $\mathrm{r}>2 \mathrm{AU}, \mathrm{N} \sim \mathrm{r}^{-4}$. This simple model predicts that most of the interplanetary dust particles is concentrated within $1 \mathrm{AU}$ in the solar system. The dust density appears to vary little between $0.7 \mathrm{AU}$ and $1.1 \mathrm{AU}$. The dust density at $2 \mathrm{AU}$ is $20 \%$ of that at $1 \mathrm{AU}$ and at $3.3 \mathrm{AU}$ it is only about $2 \%$ of that at $1 \mathrm{AU}$.

Case (b) is a very interesting model since it shows one minimum and one maximum. This case has been fitted with the radar meteor data (Southworth and Sekanina, 1973) and the result is shown as Equation 8 and in Figure 2. This model again demonstrates that for $r<0.3 \mathrm{AU}$, $\mathrm{N} \sim \mathrm{r}^{-1}$ and for $\mathrm{r}>3 \mathrm{AU}, \mathrm{N} \sim \mathrm{r}^{-3}$. The space density is minimum near $0.8 \mathrm{AU}$ and maximum at $2.4 \mathrm{AU}$.

The last case has been compared with the Pioneer 10 penetration data and is shown in Figure 3. Here again for small $r, N \sim r^{-1}$. Between $1 \mathrm{AU}$ and $3 \mathrm{AU}$ the number density

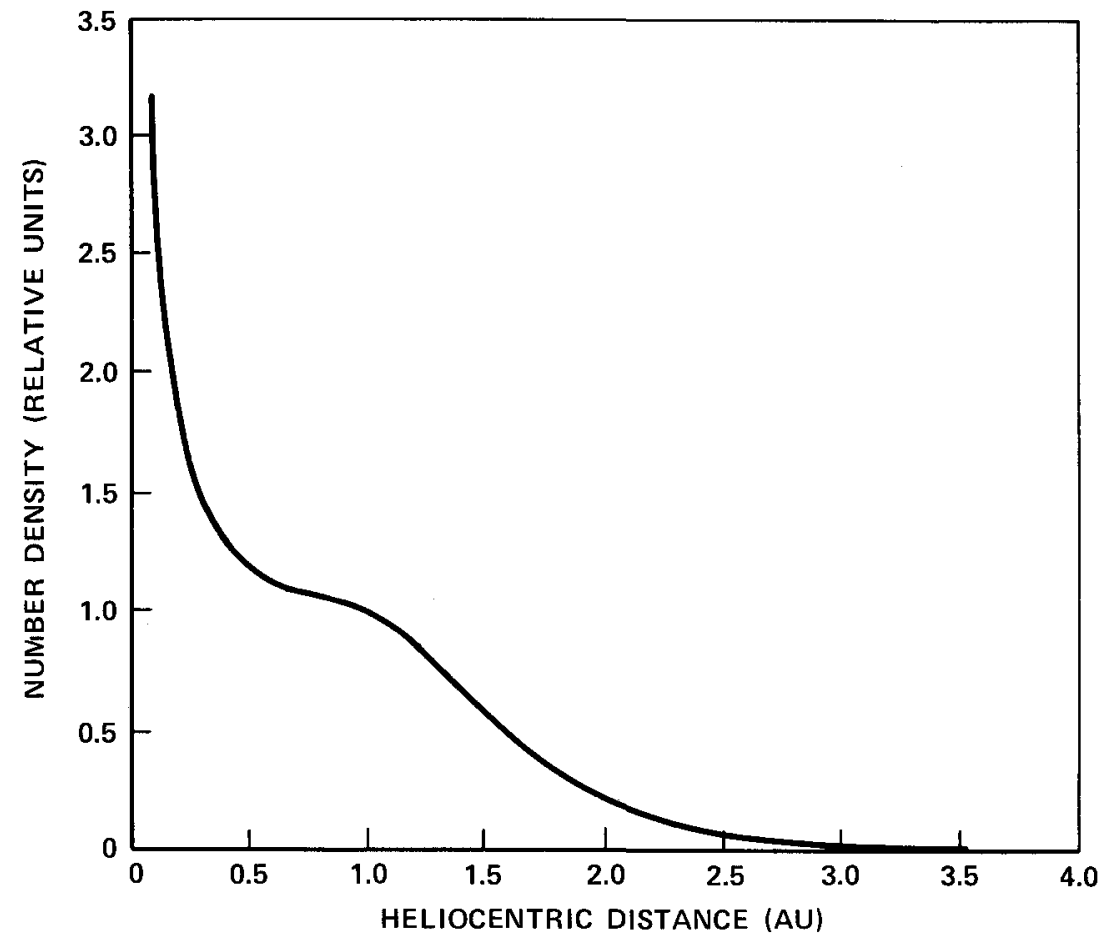

Figure 1. Radial Distribution of Zodiacal Dust 


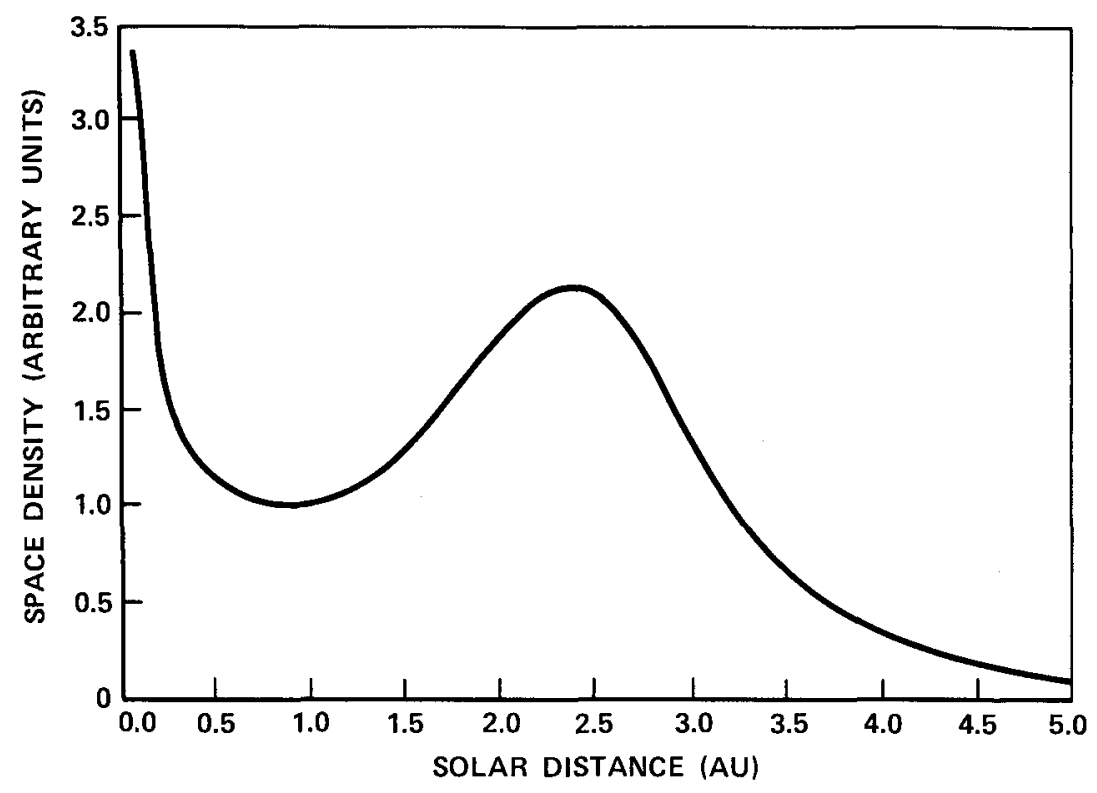

Figure 2. Space Density of Radar Meteors

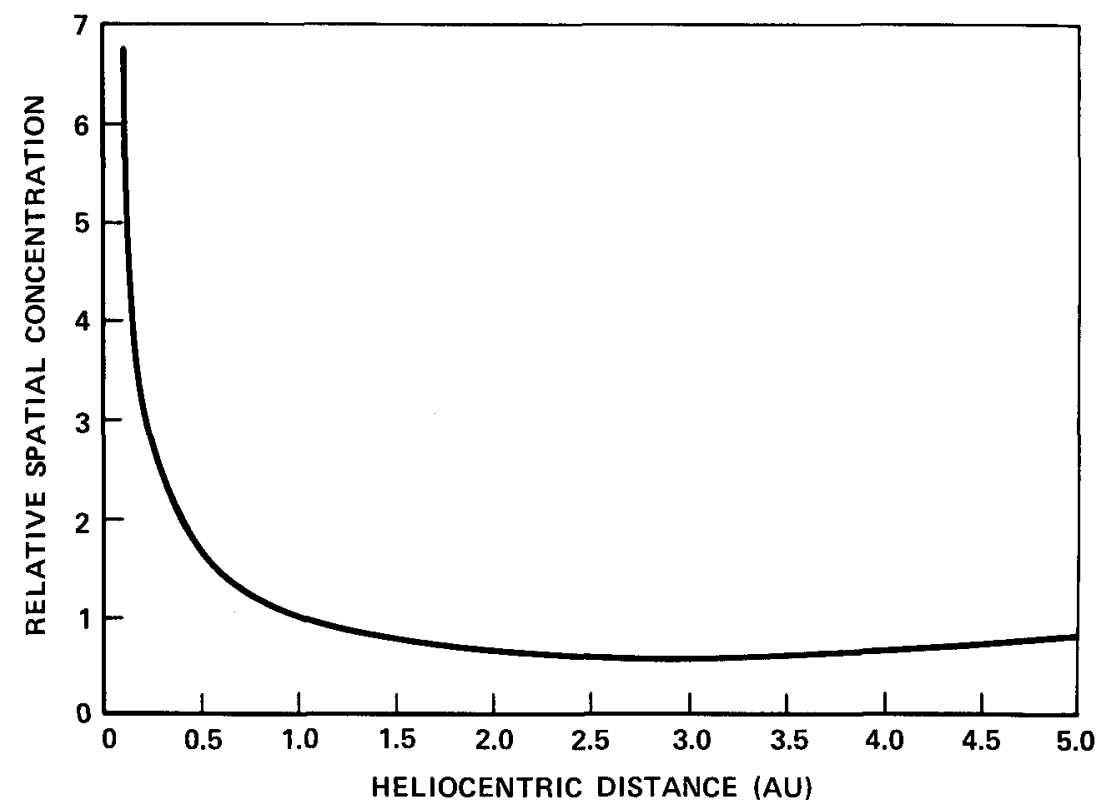

Figure 3. Spatial Concentration of Meteoroids of Mass $10^{-9} \sim 10^{-10} \mathrm{~g}$ (normalized to that at $1 \mathrm{AU}$ ) 
decreases very slowly and then starts increasing at $3 \mathrm{AU}$. The spatial concentration at $3 \mathrm{AU}$ is about $62 \%$ of that at $1 \mathrm{AU}$ and at $5 \mathrm{AU}$ it is only $80 \%$ of that at $1 \mathrm{AU}$. Thus this model predicts one minimum and no maximum in interplanetary space. It is to be noted that this model cannot explain the kind of data gap observed from the Pioneer 10 penetration measurements.

For zodiacal dust particles the spatial concentration is maximum near the sun and is essentially zero beyond $3.3 \mathrm{AU}$. The space density of larger particles such as radar meteors shows a minimum near $0.8 \mathrm{AU}$ and a maximum near $2.5 \mathrm{AU}$. For medium size particles of mass $10^{-12} \mathrm{~kg}$ and larger the number density has a minimum at $3 \mathrm{AU}$ even though the spatial variation between $1 \mathrm{AU}$ and $5 \mathrm{AU}$ is no more than $50 \%$ of that at $1 \mathrm{AU}$. In all of these cases the concentration is inversely proportional to the radial distance between $0.1 \mathrm{AU}$ and $0.5 \mathrm{AU}$.

\section{REFERENCES}

Alexander, W. M., Cosmic Dust, Science 138, 1098-1099, 1962.

Alexander, W. M., McCracken, C. W., and Bohn, J. L., Zodiacal Dust, Science 149, 1240, 1965.

Hanner, M. S., Weinberg, J. L., DeShields II, L. M., Green, B. A., and Toler, G. N., Zodiacal Light and the Asteroid Belt: The View from Pioneer 10, J. Geophys. Res. 79, 3671-3675, 1974.

Humes, D. H., Alverez, J. M., O'Neal, R. L., and Kinard, W. H., The Interplanetary and Near-Jupiter Meteoroid Environment, J. Geophys. Res. 79, 3677-3684, 1974.

Rhee, J. W., Berg, O. E., and Richardson, F. F., Heliocentric Distribution of Cosmic Dust Intercepted by Pioneer 8 and 9, Geophys. Res. Letters, 1, 345-346, 1974.

Sekanina, Z., On the Nature of the Antitail of Comet Kohoutek 1973f, Preprint No. 106, Center for Astrophysics, Cambridge, Mass., May, 1974.

Southworth, R. B. and Sekanina, Z., Physical and Dynamical Studies of Meteors, 38, NASA CR-2316, Oct. 1973. 